\title{
Meiotic nuclear restitution mechanisms in a triploid lily
}

\author{
Errico $^{1 *}$ Angela, Maria Cammareri ${ }^{2}$ and Clara Conicella ${ }^{2}$ \\ ${ }^{1}$ Dept. of Soil, Plant and Environmental Sciences, University of Naples "Federico II", Via Università 100, 80055 \\ Portici, Italy. \\ ${ }^{2}$ CNR-IGV, Research Institute of Plant Genetics, Research Division: Portici, Via Università 133, 80055 Portici, \\ Italy.
}

\begin{abstract}
Chromosome number and pollen stainability were evaluated in Lilium L. species (L. formosanum, L. miryophillum, L. regale), and in cultivars coming from Asiatic (Elite, Pollyanna, Vivaldi) and Oriental hybrid group (Cascade, Casablanca, Galilei). All the genotypes are diploid $(2 n=2 x=24)$ except cv. Elite which results triploid $(2 n=3 x=36)$. The pollen stainability ranged from 90 to $98 \%$ among diploids and from 80 to $90 \%$ in the triploid which exhibited pollen grains of different size, as well.

A detailed analysis of microsporogenesis was carried out in the triploid cultivar. The chromosome associations at diakinesis were either trivalents or bivalents and univalents. Anaphases I and II exhibited lagging chromosomes and unequal distribution of chromosomes at the two poles. Single and double bridges plus acentric fragments formed possibly due to a paracentric inversion.

Meiotic nuclear restitution mechanisms occurred following the failure of the reductional wall or, alternatively, of the equational wall. The analysis of sporads evidenced tetrads as well as dyads, triads and rare monads and poliads.
\end{abstract}

Key words: cytokinesis, Lilium, meiosis, pollen

\section{INTRODUCTION}

The genus Lilium includes approximately a hundred species classified into seven Sections (MCRAE 1998). This germplasm represents an important source of useful genes as well as a "reservoir" of allelic diversity for breeding purposes. However, gene introgression through the wide interspecific hybridization is hampered by incompatibility barriers at pre- and post-fertilization levels including $\mathrm{F}_{1}$ hybrid sterility. Nevertheless, wide hybridization in Lilium was facilitated by a complete and integrated in vitro system for pollination, fertilization and embryo-rescue (VAN Tuyl et al. 1991). To overcome $F_{1}$ sterility, somatic and sexual polyploidization approaches have been practised successfully in Lilium (KARLOv et al. 1999; LIM et al. 2001) although, the meiotic doubling offers substantial advantages over the mitotic doubling. The meiotic doubling ensures that alien chromosome segments can be transmitted since homoeologous recombination can occur during meiosis in the diploid interspe-

\footnotetext{
* Corresponding author: phone: ++39/81/2539430; fax: ++39/81/7753579; e-mail: errico@unina.it
}

cific hybrid. Indeed the occurrence of intergenomic recombination in $2 \mathrm{n}$-gametes has been clearly demonstrated in Lilium (KARLOv et al. 1999; Lim. et al. 2001) and Alstroemeria (KAMSTRA et al. 1999). Besides gene introgression, sexual polyploidization ensures the allelic diversity by influencing those characters which are dependent on intra- and interlocus non-additive interactions (Peloquin et al. 1999). If $2 n$ gametes carry non-sister or sister chromatids, they are therefore potentially heterozygous or homozygous, being considered genetically equivalent to the products of a first division restitution (FDR) or a second division restitution (SDR) (Peloquin 1983; Hermsen 1984). In this context, meiotic nuclear restitution, $2 n$-gametes and their modes of origin are crucial for breeding strategies based on wide hybridization.

In this work, a screening of Lilium genotypes for restitution events in microsporogenesis was performed through the analysis of chromosome number, pollen stainability and meiosis.

\section{MATERIALS AND METHODS}

Plant material consisted of three cultivars of Asiatic lily hybrids: Elite, Pollyanna, Vivaldi; three 
cultivars of Oriental lily hybrids: Cascade, Casablanca Galilei; three species of Lilium: L. formosanum, L. miryophillum and L. regale kindly given by Botanical Garden of Nancy (France). They were grown in an air-conditioned $\left(18-25^{\circ} \mathrm{C}\right)$ greenhouse under natural daylight at Portici (NA).

For mitotic analysis, $1 \mathrm{~cm}$ long root tips were collected, pre-treated in an acqueous solution of $0.5 \%$ colchicine for 6 hours at room temperature and fixed in ethanol-acetic acid $(3: 1)(\mathrm{v} / \mathrm{v})$ for at least $2 \mathrm{~h}$. The root tips were then hydrolized in $\mathrm{HCl} 5 \mathrm{~N}$ for $55 \mathrm{~min}$ at room temperature and stained at dark with Schiff's reagent for $2 \mathrm{~h}$ and squashed in a drop of acetic acid (45\%). After coverslip removal by freezing with liquid nitrogen, slides were made permanent by dehydration in ethanol and subsequently in xylol and then by rejoining the coverslip with Entellan (Merck, Germany).

To study microsporogenesis, $1 \mathrm{~cm}$ anthers were fixed for at least $48 \mathrm{~h}$ in ethanol-propionic acid (3:1) with a small amount of ferric chloride at room temperature and then stained with $2 \%$ acetocarmine. Slides were made permanent as abovementioned but dehydrated in butanol-acetic acid (1:1) twice and in absolute butanol once. Mature pollen grains were stained with $2 \%$ acetocarmine to estimate pollen stainability and size. In Elite, length of pollen grains and leaf stomata width were evaluated. All data were analyzed by Student's t-test with Systat Package software (WILKINSON et al. 1992).

\section{RESULTS}

Chromosome number was ascertained in Lilium genotypes analyzed in this work. The three wild species as well as the cultivars resulted diploids $(2 n=2 x=24)$ except cv. Elite which was a triploid $(2 n=3 x=36)$. In the triploid, the values of leaf stomate size (width/length) increased significantly $(\mathrm{P} \leq 0.001)$ in comparison to diploid cultivars $(0.78 \pm 0.03$ vs. $0.51 \pm 0.03)$. The pollen stainability ranged from 90 to $98 \%$ among diploids and from 80 to $90 \%$ in the triploid. As concerning the pollen size, a larger variability characterized the pollen size in Elite as compared to the diploids. Pollen grains observed in diploid cv. Pollyanna from Asiatic group showed a length of 85-90 $\mu \mathrm{m}$ and have been considered as the standard. Based on the length of pollen grains, pollen of Elite was grouped in two classes: A $(\leq 90 \mu \mathrm{m})$ and B $(95 \mu \mathrm{m})$. The latest class occurred at a frequency of $20 \%$ and possibly included both pollen with chromosome number higher than $x=12$ and $2 n$ pollen.

The microsporogenesis analysis performed on the diploids did not evidence any abnormality in Pollen Mother Cells (PMCs). In diakinesis, the chromosome pairing was normal with occurrence of 12 bivalents. At anaphases I and II, the disjunction was normal and only tetrads formed following a successive type cytokinesis occurring after telophase I and II. In the triploid, the chromosomes arranged as trivalents or as bivalents and univalents at diakinesis (Fig $1 \mathrm{~A}$ and $1 \mathrm{~B})$. The

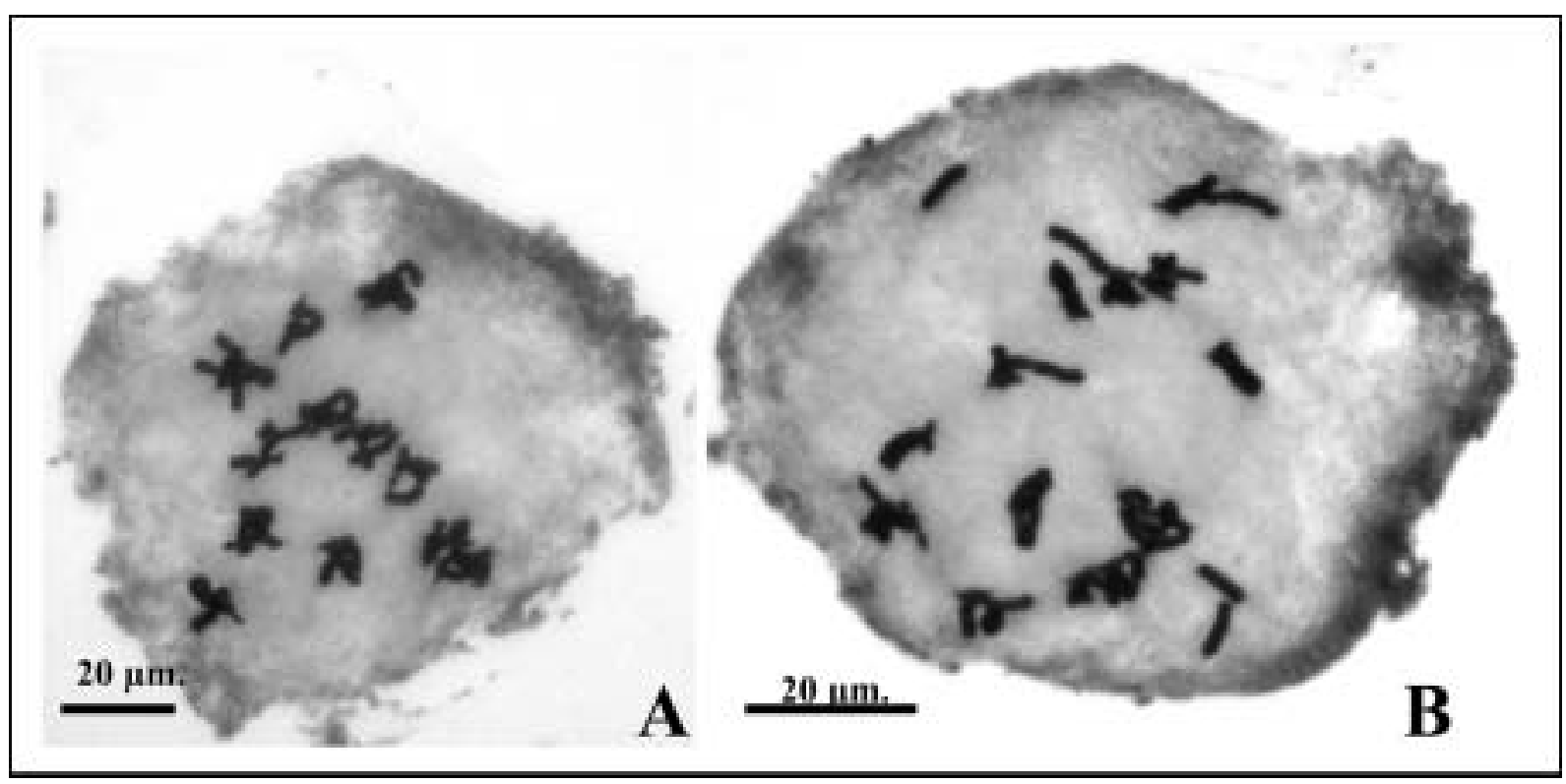

Fig. 1 - Chromosome associations at diakinesis in Elite. A: 12 trivalents. B: 9 trivalents +3 bivalents +3 univalents. 
average number of associations per PMC (the range is reported in brackets) was as following: 9.5 (7-12) trivalents, 2.4 (0-6) bivalents and 1.5 (0-3) univalents. Anaphases I and II exhibited lagging chromosomes and unequal distribution of chromosomes at the two poles (Fig. 2A). Table 1 reports the frequency of anaphases and telophases with bridges and fragments at meiosis I and II. Bridges and/or fragments were absent in only $12 \%$ of PMCs at anaphase I and $30 \%$ at telophase II. A single bridge occurred at a frequency of $44 \%$ and $48 \%$ in anaphase I and II, respectively (Fig. 2A). In addition to bridges and fragments, anaphases and telophases exhibited also lagging chromosomes depending on the univalents with a frequency of $37 \%$ and $52 \%$, respectively (Figs 2B, 2C and 2D). Asynchrony in the meiotic stages between the two cells in the meiocyte was also observed: figure $3 \mathrm{~A}$ reports the case of one cell at metaphase II and the other at anaphase II.

As concerning the cytokinesis, in some PMCs a failure of the reductional wall (Fig 3B) or, alternatively, of the equational wall in one of the cells (Fig. 3C) was observed. Following this failure, nuclear restitution events were observed at the end of meiosis (Fig. 3D) giving rise to sporads with $2 n$ microspores such as triads (5\%) and dyads (Figs
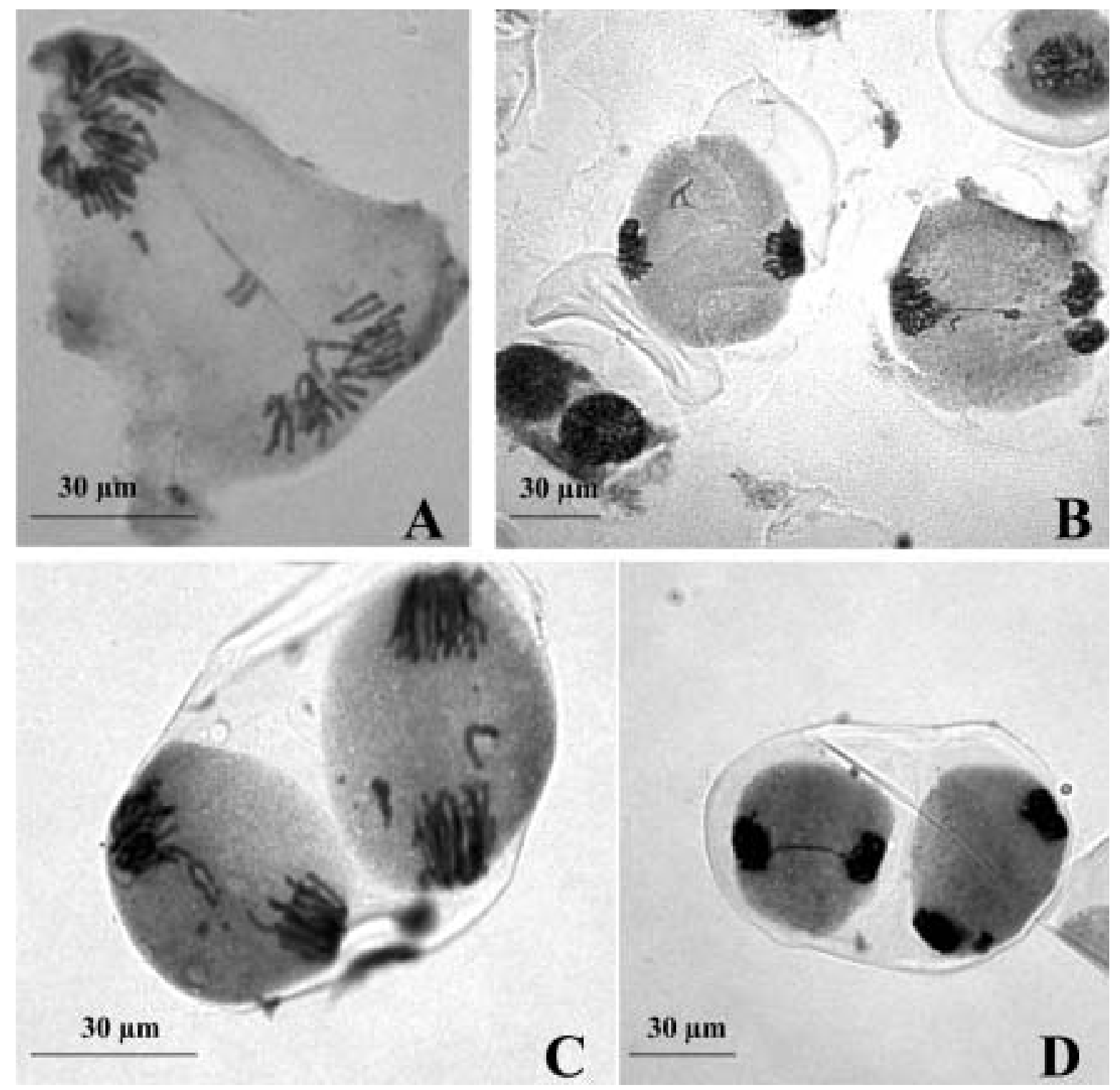

Fig. 2 - Behaviour of the univalents and the paracentric inversion in meiosis of Elite. A: anaphase I with a single bridge, a fragment and unequal distribution at the two poles. B: lagging chromosomes, fragments and bridge at telophase I. C: lagging chromosomes and fragments at anaphase II. D: lagging chromosomes and bridge at telophase II. 
Table 1 - Frequency of anaphases and telophases with bridges and/or fragments at meiosis I and II.

\begin{tabular}{|c|c|c|c|c|c|c|c|c|c|c|c|}
\hline \multirow{4}{*}{ Meiotic Stage } & \multirow{4}{*}{$\begin{array}{c}\text { PMCs } \\
\text { analyzed } \\
\text { n. }\end{array}$} & \multicolumn{10}{|c|}{$\%$ of PMCs } \\
\hline & & \multicolumn{4}{|c|}{ with no bridge } & \multicolumn{3}{|c|}{ with one bridge } & \multicolumn{3}{|c|}{ with two bridges } \\
\hline & & \multirow{2}{*}{$\begin{array}{c}\text { no } \\
\text { fragment }\end{array}$} & \multicolumn{3}{|c|}{ fragment } & \multirow{2}{*}{$\begin{array}{c}\text { no } \\
\text { fragment }\end{array}$} & \multicolumn{2}{|c|}{ fragment } & \multirow{2}{*}{$\begin{array}{c}\text { no } \\
\text { fragment }\end{array}$} & \multicolumn{2}{|c|}{ fragment } \\
\hline & & & 1 & 2 & 3 & & 1 & 2 & & 1 & 2 \\
\hline Anaphase I & 92 & 12 & 26 & 3 & 2 & 16 & 28 & - & - & 13 & - \\
\hline Telophase I & 172 & 17 & 21 & 10 & 3 & 12 & 26 & 3 & 3 & - & 5 \\
\hline Anaphase II & 57 & 19 & 28 & 3 & - & 19 & 29 & - & - & - & 2 \\
\hline Telophase II & 47 & 30 & 34 & 6 & - & 4 & 26 & - & - & - & - \\
\hline
\end{tabular}
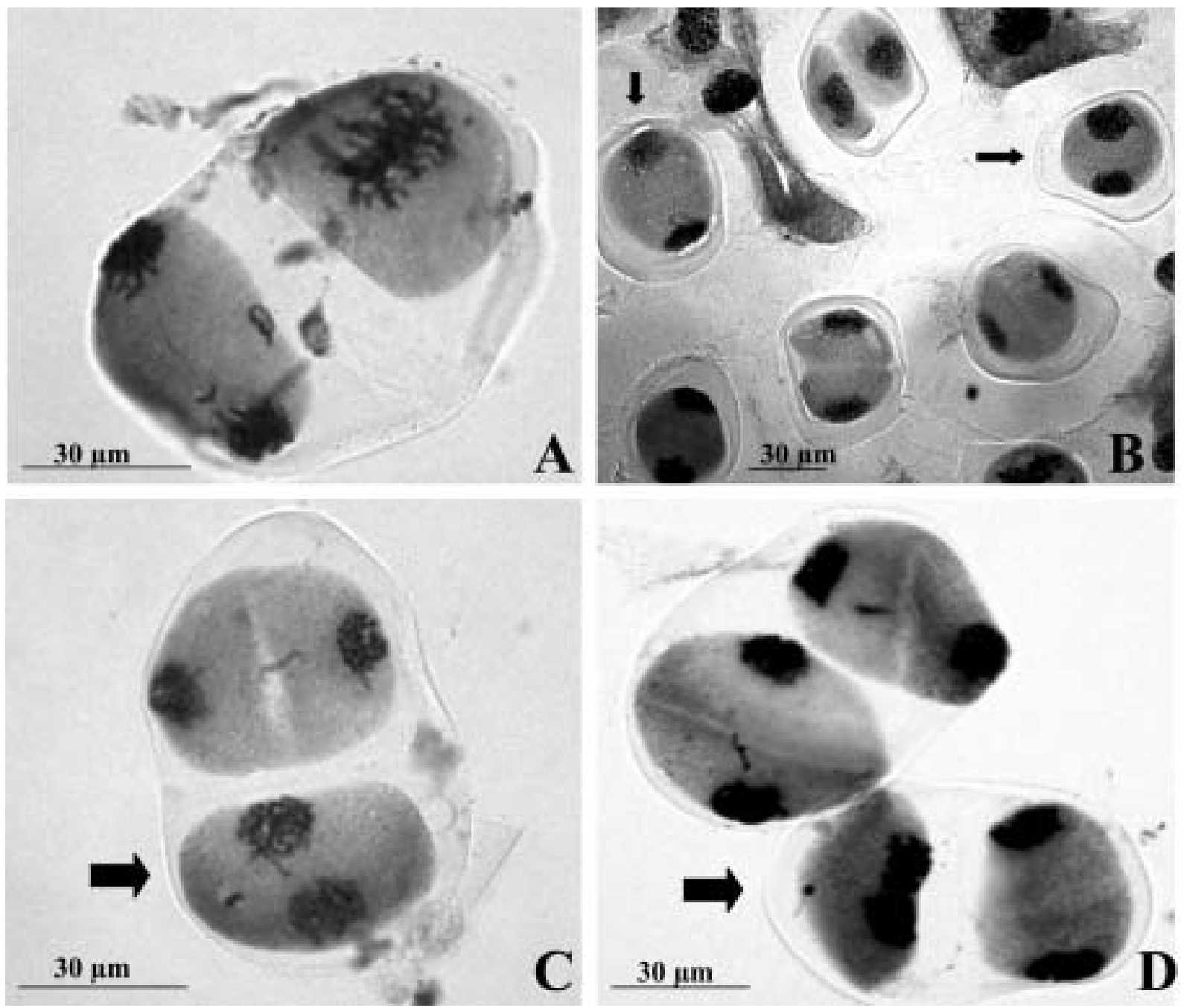

Fig. 3 - Asynchrony and meiotic nuclear restitution events. A: PMC with one cell at metaphase II and the other at anaphase II. B: telophase I showing absence of cytokinesis (arrows). C: PMC at telophase II with absence of cytokinesis in one of the two cells and nuclei irregularly positioned (arrow). D: two nuclei very close in the cell (arrow).

4A and 4B). Rarely, cytokinesis was absent at all giving rise to a monad (Fig.4C). The occurrence of univalents and the absence of restitution events gave rise to poliads. Although the tetrad was the most abundant class, the microsporogenesis in Elite resulted quite perturbed. In fact, lagging chromosomes, fragments and different nuclear size occurred in $33 \%$ of the tetrads. 


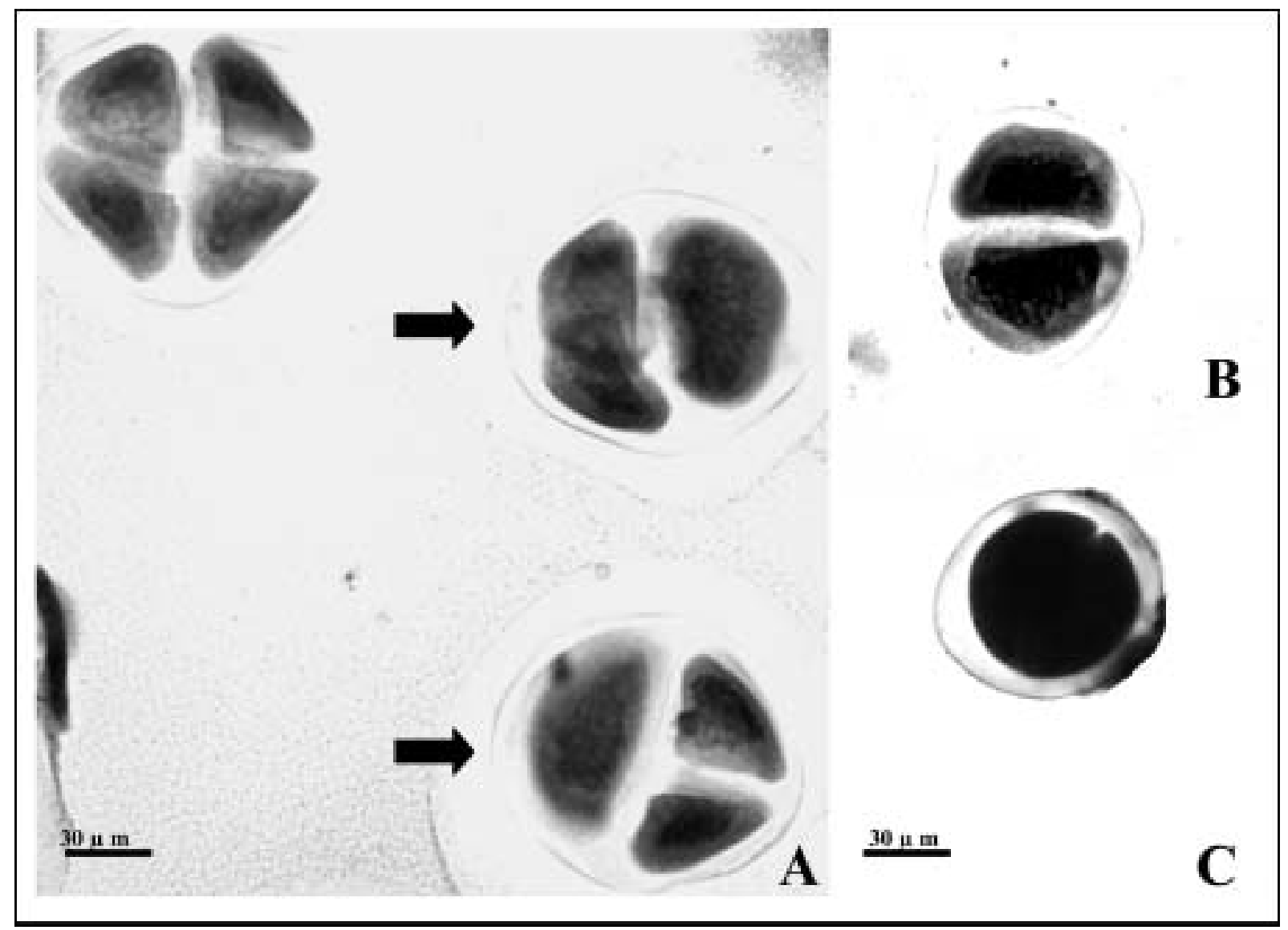

Fig. 4 - Sporad stage. A: one tetrad and two triads indicated by arrows. B: one dyad. C: one monad.

\section{DISCUSSION}

All the genotypes analyzed in this work are diploid $(2 n=2 x=24)$ except the triploid variety Elite $(2 n=3 x=36)$ belonging to Asiatic hybrids. It is reported that about $70 \%$ of Lilium species are diploid whereas triploidy occurs spontaneously in L. lancifolium (tiger lily) (NoDA 1991), L.medeoloides (SAMEjIMA 1958) and L. bulbiferum (NodA and Schmitzer 1993). In the latest species, the occurrence of autotriploidy in a natural diploid population is explained with the fertilization between an haploid egg cell and a $2 n$ pollen grain (NodA and Schmitzer 1993). Triploids have been also obtained from backcrosses such as Asiatic hybrid x (L. longiflorum x Asiatic hybrid) (ALA), Oriental hybrid $\mathrm{x}$ (L. longiflorum $\mathrm{x}$ Asiatic hybrid) (OLA) (KArLov et al. 1999). In particular, ALA triploids originated from unilateral sexual polyploidization based on $2 n$ pollen produced by the diploid interspecific hybrids (Lim et al. 2001). The frequency of triploids increases in breeding material since the dropping in pollen fertility occurring in interspecific hybridization is overcome by the formation of $2 n$ pollen which is generally the only viable one (LIM et al. 2001).

Based on restitution mechanisms evidenced in microsporogenesis in this work, it can be hypothesized that triploid Elite could be originated from unilateral sexual polyploidization. Infact, $2 n$ pollen occurred in Elite due to cytokinesis failure either at meiosis I or meiosis II with genetic consequences equivalent to first division restitution (FDR) or second division restitution (SDR), respectively. Previously, in Lilium LA hybrids, FDR, SDR and a novel type of restitution mechanism named Indeterminate Meiotic Restitution (IMR) were detected as responsible of $2 n$ pollen formation (LIM et al. 2001). Meiotic nuclear restitution events can depend on various abnormalities occurring during different meiotic steps such as chromosome pairing, chiasma formation, spindle orientation at metaphase II, cytokinesis and cell wall formation. The well known meiotic mutations described for $2 n$ gam- 
etes in Solanum species were parallel or fused spindles in microsporogenesis and omission of second division in macrosporogenesis (MoK and Peloquin 1975; Ramanna 1979; Werner and Peloquin 1987). However, the abnormalities of microsporogenesis described in Solanum that shows a simultaneous type cytokinesis are different from Lilium those ascertained in that has a successive cytokinesis typical of monocotyledonous.

The occurrence of meiotic restitution mechanisms evidenced in this work in Elite guarantees the formation of balanced gametes which contribute to increase the pollen fertility. However, unbalanced pollen of Elite seems equally fertile as evidenced by the pollen stainability analysis. Similarly, in allotriploid crops such as banana, plantain and peanuts (SHEPHERD 1999) balanced haploid $(x)$, diploid $(2 x)$ and triploid $(2 n)$ gametes were produced at a high frequency.

As concerning other features of the triploid, Elite showed also a size increase not only for pollen but also for guard cells which are positively correlated to ploidy levels in Lilium as reported by McRae (1987).

Besides the meiotic restitution mechanisms, Elite showed also other aberrations in microsporogenesis: 1. a partial pairing failure which caused a high number of univalents behaving subsequently as laggards; 2 . bridges and fragments at anaphases. The pairing behaviour is obviously depending on the existence of three genomes in Elite. Although the origin of Elite is unknown, L. lancifolium and L. bulbiferum, abovementioned as triploids, are reported to be parental species of Asiatic hybrids (McRAE 1998). Bridges and fragments evidenced in Elite could depend on a paracentric inversion. It is known that crossing over within the paracentric loops alone or with a crossover in the interstitial segment leads to the appearance of chromatin bridges and fragments at anaphase I and II depending on dicentric chromatids and acentric fragments (PALMER 2000).

In conclusion, it can be suggested that the cytological screening like that performed in this work on different genotypes of Lilium should be usually done before starting a breeding programme. Moreover, the identification of restitution mechanisms reinforce the importance of $2 n$ gametes for Lilium breeding.

Contribution n 100 from DISSPA, Dept. of Soil, Plant and Environmental Sciences: n 64 from CNR-
IGV, Research Institute of Plant Genetics, Research Division: Portici.

\section{REFERENCES}

Hermsen J.H.TH., 1984 - Mechanisms and genetic implication of $2 n$ gamete formation. Iowa State Journal of Research, 58: 421-434.

Kamstra S.A., Ramanna M.S., De Jeu M.J., Kuipers A.G.J. and Jacoben E., 1999 - Homologous chromosome pairing in the distant bybrid Alstroemeria aurea $\times A$. inodora and the genome composition of bakcross derivatives determined by fluorescent in situ bybridisation with species-specific probes. Heredity, 82: 69-78.

Karlov G.I, Khrustaleva L.I., Lim K.B. and VAN TuIL J.M. 1999 - Homologous recombination in $2 n$-gametes producing interspecific bybrids of Lilium (Liliaceae) studied by genomic in situ bybridization (GISH). Genome, 42: 681-686.

Lim K.B., Ramanna M.S., Dejong J.H., Jacobsen E. and Van Tuyl J.M., 2001 - Indeterminate Meiotic Restitution (IMR): a novel type of meiotic nuclear restitution mechanism detected in interspecific lily bybrids by GISH. Theor. Appl. Genet., 103: 219230.

McRAe J., 1987 - Stomata size E Pollen Characteristics as an indicator of chromosome number in lilies. NALS-LYB, pp. 19-26.

McRAe E.A., 1998 - Lilies: A Guide for growers and collectors. Timber press Portland Oregon USA.

Mok D.W.S., and. Peloquin S.J., 1975 - Three mechanisms of $2 n$ pollen formation in diploid potato. Can. J. Genet. Cytol., 17: 217-225.

Noda S. and Tsuchiya T., 1991 - Chromosomal variation and evolution in the genus Lilium. In, Chromosome engineering and their distribution in plants: genetics, breeding, evolution, Elsevier, Amsterdam. Ed: Gupa P.K: pp: 507-524.

Noda S. and Schmitzer E., 1993 - Natural occurrence of triploid Lilium bulbiferum, native to Europe. Lily yearbook, 43: 78-81.

Palmer R.G, Sun H. and Zhaom L.M., 2000 - Genetics and Cytology of Chromosome inversion in Soybean Germoplasm. Crop Sci., 40: 683-687.

Peloquin S.J., 1983 - Genetic engineering with meiotic mutants. In: D.L. Mulcahy and E. Ottaviano (Eds), Pollen Biology and Implication for Plant Breeding, 311-316.

Peloquin S.J., Boittau l. S. and Carputo D., 1999 - Meiotic mutants in potato: valutable variants. Genetics, 153: 1493-1499.

Ramanna M.S., 1979 - A re-examination of the mechanism of $2 n$ gamete formation in potato and its implication for breeding. Euphytica, 28: 537-561.

SAMEJIMA J., 1958 - Meiotic behavior of accessory chromosomes and their distribution in natural population of Lilium medeoloides A. Gray. Cytologia, 23: 159-179. 
SHEPHERD K., 1999 - Cytogenetics of the genus Musa. International Network for the Improvement of $\mathrm{Ba}$ nana and Plantain. Montpellier, France. IPGRI, Rome, pp 1-157

Van Tuyl J.M., Van Dien M. P., Van Creij M.G.M., Van Kleinwee T.C.M., Franken J. and Bino R.J., 1991 - Application of in vitro pollination, ovary culture, ovule culture and enbryo rescue for overcaming incongruity barriers in interspecifiv lilium crosses. Plant Sci., 74: 115-126.
Werner, J.E., and Peloquin S.J., 1987 - Frequency and mechanisms of $2 n$ egg formation in haploid tuberosum-wild species F1 bybrids. Am. Potato J., 64: 641-654.

Wilkinson L., HiLl M.A., and VANG E., 1992 - SYSTAT: Statistics. Version 5.2 - SYSTAT, Inc..

Received III.08.2005; accepted X.10.2005 